Research Note

\title{
Preforms: Golden Nuggets of Language - A Brief Introduction to a Speculative Monograph
}

Clyde Coreil

New Jersey City University

2039 Kennedy Blvd,

Jersey City

New Jersey, USA

Corresponding author: coreil@rcn.com

\section{About the author}

Clyde Coreil received a Master of Fine Arts from Carnegie Mellon University in Pittsburgh. He received his Ph.D. in linguistics from the Graduate Center of The City University of New York. His dissertation, Fusion in Language: A Case for Supralexical Units, received the 1992 Edward Sapir Award from The New York Academy of Sciences. Although Dr. Coreil has replaced "Supralexical Units" with the word "Preformulations," his life-long interest in this field of study has continued unabated. Clyde Coreil has written some 20 plays, one of which received a playwriting prize from the National Endowment for the Arts, given by the USA government. He has also written the music and lyrics of some 30 country songs. 


\section{Instant of Preformulation}

Language structures-such as "by the way," "go-to guy" and "day in and day out"-are usually dismissed and/or ignored as spurious fixed phrases that are of little importance to syntax and semantics. In this speculative monograph, I wish to examine the possibility that these "Preforms" are the most important configurations in any language: in effect, that they can be said to be at the pinnacle of the linguistic hierarchy. ("Pre-" because when speakers spoke, as Allison Wray points out, they had already fixed this "form" in their minds.) It is my hypothesis that all Preforms are based on the speaker's having consciously or subconsciously committed to memory an enormous number-say 50,000-of fixed phrases or stretches of lexical items. On speaking, he or she incorporates these stretches or "Preforms" into the syntax of his or her speech. Among the main ramifications of my hypothesis seems the probability that there comes to be an instant of Preformulation at which these lexical items immediately becomes fixed and frozen rock solid-except sometimes for tense, number and person.

The manner in which lexical items come together in such a way that they can undergo this transmutation and become Preforms would seem to be of enormous significance to linguistics. A case could be made that the unitary nature of Preforms has parallels in virtually all species of living things. The control of fixed variations in the songs of birds, the barking and growls of dogs, the buzzing calls of cicadas, katydids, and crickets-all of these are forms of communication, as are the vastly more complex different Preforms. But all seem to have at their core-an unchangeable element.

Praiseworthy efforts have been made to assemble a limited number of syntactic principles that would govern an unlimited number of relationships within the lexicon. These principles, however, have not included the progression that leads to the remarkable instant of transmutation that we are calling "Preformulation." If this is accurate, it could have widespread implications-perhaps none as relevant as for the teaching of second languages. Here, students might be assigned to memorize verbatim five or ten Preformed phrases or "Preforms" for each class session. (Examples of 100 Preforms are given below.) The teacher would then discuss these Preforms and put them in sentences. After a few sessions, the students would have access to 20 or 30 stretches of words that have absolutely no mistakes inside their boundaries-that is according to English as it is spoken by Fully Native Speakers. Preforms are "stepping stones to help you cross the lake," as one of my students put it.

By the end of the term, students would have fixed in their memories, more than 500 Preforms. This seems to be a truly excellent way to study grammar-through the concrete examples of actual words, not the study of principles or rules. Presently, it seems to be hoped that awareness of the behavior of different classes to which individual words belong is the closest we can come to a generative framework. I reject this notion that close linguistic analysis is the key: I would strongly suggest that the de facto unlimited memory of specific Preforms opens the path to successful speech. That is my fundamental hypothesis.

In these introductory pages, I will briefly consider Preforms-short for "Preformulations"- in terms of 21 specified characteristics. The 21 characteristics of Preforms are the following:

Journal of Management and Training for Industries, Vol.8, No.1, 2021 
(1) Formed Instantaneously;

(2) Fixed and Frozen;

(3) Endures Indefinitely,

(4) Do Not Accept Change or Modification;

(5) Often an Aberration of Syntax;

(6) 50,000+ in General English;

(7) 500,000 in Specializations;

(8) Irreversible Union;

(9) Socially Constructed;

(10) No Previous Existence;

(11) New Semantic Feature;

(12) Dependent on Memory;

(13) Teachable, and Learnable;

(14) Articulates the Otherwise Inexpressible;

(15) Helps a Language become Itself;

(16) Helps Conceive and Express with Great Precision;

(17) Constitutes an Index of Verbal Dexterity;

(18) Acquiring Them Can Be a Life-long Process;

(19) Unending Generation;

(20) Provides an Entry-Way into a Given Culture;

(21) Constitute much of that Given Culture.

The result of these observations will, I think, support our initial suggestion that the Preform is the single most important structure in linguistic theory. Because it is eminently learnable, it might just revolutionize the teaching of second languages-at least, it might provide a different approach.

\section{Examples of Preforms}

I consider Preforms to be at the pinnacle of verbal language. What might support this seemingly outrageous claim is the estimate of 50,000+ Preforms that are to be found in General English, to say nothing of Medical, Legal and Scientific specializations-and just possibly in a great many other parts of verbal languages. I present some 100 Preforms immediately below, and more at the end of Chapter Two of this book. Having suggested the pinnacle proposition, it is incumbent upon me to present some hard and fast examples of the Preforms. It is also proper that I base my claim on my experience as a Fully Native Speaker of English, as a teacher of English as a Second Language, and as a holder of the Ph.D. degree in Linguistics (not Applied Linguistics) which I earned in 1992 at the Graduate Center of The City University of New York. I present here a tiny fragment of the memorized structures which I am calling "Preforms." 
Table 1: 100 Examples of Preforms

\begin{tabular}{|c|c|c|c|}
\hline 1 & lock, stock and barrel & 51 & over and over again \\
\hline 2 & give me a break & 52 & plunge into despair \\
\hline 3 & hand-in-hand & 53 & If I were you, I would. \\
\hline 4 & give me a hand & 54 & woven into the very fabric \\
\hline 5 & at wit's end & 55 & in this day and age \\
\hline 6 & All's well that ends well. & 56 & the way in which $\cdots$ \\
\hline 7 & That's neither here nor there. & 57 & rub me the wrong way \\
\hline 8 & get one's goat & 58 & lose all hope \\
\hline 9 & head over heels in love & 59 & Hope springs eternal. \\
\hline 10 & ump-teen (many) & 60 & day in and day out \\
\hline 11 & identity crisis & 61 & playing hard to get $\cdots$ \\
\hline 12 & fit as a fiddle & 62 & Spring has sprung. \\
\hline 13 & flat as a pancake & 63 & Skip to my Lou, my darlin'. \\
\hline 14 & Man Friday & 64 & bright-eyed and bushy-tailed \\
\hline 15 & in the closet & 65 & nothing-burger \\
\hline 16 & reach for the sky & 66 & yelling "Fire" in a crowded theater \\
\hline 17 & line his pockets & 67 & closing time \\
\hline 18 & run out the clock & 68 & They are about to close. \\
\hline 19 & All hell will break loose. & 69 & She had an hour-glass figure. \\
\hline 20 & with eyes wide open & 70 & Don’t cross him. \\
\hline 21 & the kindness of strangers & 71 & Do not look a gift horse in the mouth. \\
\hline 22 & right as rain & 72 & Heaven knows what he will do. \\
\hline 23 & born in the USA & 73 & dead in the water \\
\hline 24 & come in on a wing and a prayer & 74 & sleep-walking \\
\hline 25 & chopping block & 75 & The buck stops here. \\
\hline
\end{tabular}




\begin{tabular}{|c|c|c|c|}
\hline 26 & hand it up & 76 & ...for which there is no cure \\
\hline 27 & The writing is on the wall. & 77 & ...from which there is no escape \\
\hline 28 & look alive & 78 & ...in which there is no hope \\
\hline 29 & by virtue of the authority vested in me & 79 & by the way \\
\hline 30 & mind-boggling & 80 & That's all I have to say about that. \\
\hline 31 & just like that & 81 & hit the spot \\
\hline 32 & There, but for the grace of god, go I. & 82 & The fact of the matter is that. \\
\hline 33 & cherry-pick & 83 & What's the matter with him? \\
\hline 34 & smoking gun & 84 & Call a spade, a spade. \\
\hline 35 & $\operatorname{dig}$ up dirt on $\cdots$ & 85 & all of the above \\
\hline 36 & send a signal & 86 & an 800 -pound gorilla in the room \\
\hline 37 & The gun had a hair trigger. & 87 & all by myself \\
\hline 38 & Let's hit the hay. & 88 & et awa with murder \\
\hline 39 & Give them their money's worth. & 89 & fire broke out \\
\hline 40 & do a number on $\cdots$ & 90 & nod of approval \\
\hline 41 & show him a thing or two & 91 & that being so \\
\hline 42 & sow one's wild oat & 92 & make me an offer \\
\hline 43 & Don't feel all that well. & 93 & easier said that done \\
\hline 44 & might well & 94 & He has his ducks in a row. \\
\hline 45 & wait in the wings & 95 & worst nightmare \\
\hline 46 & stop on a thin dime & 96 & stick to her like glue \\
\hline 47 & lo and behold & 97 & Chips fall where they may. \\
\hline 48 & killing machine & 98 & one of the crowd \\
\hline 49 & break bread with & 99 & the jury is still out \\
\hline 50 & hidden in plain sight & 100 & go-to-guy \\
\hline
\end{tabular}


Mastering syntax leads to a doctorate in the internal structure of language, the dark underbelly that is thought common to all languages, and is only distantly related to learning a particular second language. Possibly, the mistake was for non-syntacticians to assume that the knowledge, methods and theories of syntacticians was superior to their own. That is about the time that a few of the Non-Syntax folks started hanging around linguistics departments, sometimes hoping that the thinking of the latter group would rub off on them. It is reminiscent of the study of Art History and the study of Studio Art. The relation between the two is tenuous at best. Art Historians are concerned only with the philosophical and social locus of all things concerning the visual and tactile arts (as compared to, say, music). Their main motivation seems to be to uncover Permanent Truths. The Studio Artist, on the other hand, is primarily absorbed in the ongoing process of making paintings, drawings, sculptures and countless other activities of the Present Moment.

Be it fish or fowl-I stumbled onto what is little more than a simple observation of the instantaneous and radical changes that occur at the very moment that a phrase becomes a Preform. That's it: at first, nothing more. I am also interested in the elusive reason why so little of the second language is retained-and just possibly even learned in the first place. The way in which the human being works (as far as I can tell) is that the mind just keeps on churning, no matter what. Somewhat predictably, the simple. The original seed has grown and turned into a hopefully unified Theory of Preformulation that I present in greater detail in the subsequent parts of this book. I kept telling myself that the central arguments should be presented succinctly in the introduction. But I didn't know exactly what those arguments were. Now, I think I do.

I am amazed that Preforms were as little studied as they are, and that their role in language would still be in the cauldrons of little-known brews. That lack of awareness has led directly to the deficiency of progress among most second language students-seemingly everywhere. With a curious mixture of linguistic theory, education, and psychology, these classes generally came up with students who could barely assemble even one of the 100 Preforms cited above. Something was-possibly is-wrong in the state of Denmark. In my humble opinion, what was sorely missing is (1) the concept of the Preform and (2) a desperate stress on the need for memorization.

I advocate teachers' listing of the Preforms in the languages they teach, and reorganizing their curricula around this list. That is a lot to ask, but it is in close keeping with what I am describing in this paper. Do that, and I think that your students will steal a march on all who are studying grammar with the hope that one day they will learn enough to ask in the second language, "Where can I find a hot dog?"

\section{New Semantic Feature}

None of the major words in any of the 100 examples of Preforms above ever change. For example, "line his pockets" (17) means to steal money at work. I can certainly say "line her pockets" or "line their pockets" but I can't say "*She lined her coat in her new job." If I do say that, I lose the meaning of "steal" and refer only to her adding some cloth to the coat. This structure-"line his coat"-is perfectly grammatical, but the notion of "steal" is missing. It is the special additional semantic feature that was added when "line his pockets" became a Preform. All Preforms have such a feature-or the narrowing to one of many semantic 
variables. Before, things were normal: when you talked about linings in clothes, you talked about warmth, fabric, shape, buttons and the like. After Preformulation, virtually none of these were referenced. Notions shifted to things like "honesty," "wealth" and "deceit." This was, indeed, it seems, from another kind of language!

If there were only forty or fifty such usages, we could dismiss them, saying that all grammars leak. But when that number approximates 50,000+, that old explanation seems wanting. It would appear that something else is at work here. I think that the unknown process at work is a sudden transmutation of the phrase to the Preform. I suggest that this change of form is caused by the ultimate "Zap"-in which the phrase instantaneously undergoes an irreversible union into a particular Preform-complete with a singular semantic feature that did not exist before. This characteristic-No Previous Existence-is part of what makes the particular Preform unique. Coupled to the qualities cited above, Singular Semantic Feature and Irreversible Union seem to come into their own. The latter refers to the fact that Preformulation is a one-way street and cannot be reversed. Un-Zapped stretches of words seem to arrange themselves in patterns of different proximity to one another. These patterns often increase in some of the values that would be acquired if the ultimate point of the Preform were to be blazingly reached. This presently mysterious Preformulation seems to be the highest goal in language, one that appears to have received no critical attention whatsoever.

Also, in the Preform, there was a sudden "freezing" of structure. Before, you could employ the passive, virtually at will, with the ability to go from "She made a dress," to "A dress was made by her." Such a perambulation was quite useful if you did not wish to specify the agency or person doing something: "The funding for the poor was drastically cut." We don't have to say who cut the funds. Such became filled with dense clouds instead of clarity with our new aberrant structure: "*His pockets were lined because of greed." This is a somewhat baffling sentence. Why? Because of the attempt to change the voice of the transitive, active Preform-"He lined his pockets with"-to the passive-"*His pockets were lined with." Such generic changes are de facto impossible in Preforms.

I capitalize "Preform" because no word comes to mind for the full concept in linguistics. Part of the concept is the prohibited loss of the possibility of going from one voice (here "active") to the other (here "passive") within the boundaries a particular Preform. At the instant of this particular "Zapping" or "Preformulation," the alteration was extensive and included the feature of "voice."

\section{Deviated Syntax}

As the number of Preforms approximates-50,000+-we should re-think fundamentals. Although few scholars refer to Generative Grammar these days, it was-until fairly recently-thought possible to locate most if not all elements of a sentence on a symbolic chart of syntax and thereby approve the generation of a new sentence. That is blown out of the water by the huge number of fixed Preforms, many of which have the very aberrations of syntax as part of their identity: "go for broke," "in harm's way," "seize the day," "ride shotgun," "long in the tooth," and "do a number on." We are faced with a new possibility. Did syntax tell the Preforms how NOT to shape themselves in order to enhance their status as being unique and requiring a 
memory that is powerful beyond measure? Often, that does indeed seem to be the case. For example, right on the money and I kid you not.

It also points to a central role of the memory-both verbatim and otherwise-in verbal language. I think that the role of memory in the generation of abstract sentence structures as well as realized sentences is vastly underestimated at present. In my opinion, the proliferation of Preforms is evidence that elaborate syntactic formulas are-by and large-superfluous in the generation of language. In an attempt to compensate for the assumed limitations of memory, we have done ourselves great harm. In other words, we have sought to solve a problem that does not really need solving, and ignored others that do.

\section{Uber-Intelligent Homunculus}

Be that as it may, there does seem to be present some kind of impersonal homunculus or "Uber-Intelligence" that never operates to our personal service or disservice, but always presents or discusses a situation in purely objective terms. For example, take the notion that syntax can locate a part of itself from which it can deviate in seeking uniqueness. This implies a consciousness, seemingly within the language-generating/perceiving faculty itself-in other words, a homunculus-like entity. Thoughts related to this "homunculus" seem to have been minimized upon the development of the microscope, which revealed apparent differences in shape and form. What still seems to be with us, however, is the structural and psychic similarities between us and our elusive, companion, the homunculus.

You might point out that "line his pocket" is a figure of speech and that is why the meaning changed. Exactly: you're right! That is to say that "steal" is precisely the meaning that was added at the instant the group of words-"line his pockets"-became a Preform. That added semantic feature is part of what Zaps the group of words-or "stretch"-into a Preform, a unique language structure that-once made-can never go back to the state of being simply an "un-zapped" phrase. You can't "*Un-Preform" a "Preform." It's a one-way process.

It's the same with all of the 100 other Preforms listed above. My reference to "50,000+" is an unverified estimate. This figure, however, seems to me to be conservative, judging from the structures cited; from the several hundred that I present in my websites Formulaics.com and CoreilImagination.com; and from my many notes of Native speech. There could be far more than 500,000 Preforms in all of the specializations of English. None of these basic structures can ever change: they are always the same, which is what makes them most welcome. They are like nuggets of gold: hard as rock, impermeable and valuable.

Preforms are valuable because-within their boundaries-they never need or tolerate the slightest reduction or alteration in sequence, prepositions, nouns, verbs or adjectives. Often, even punctuation, pronunciation, and stress remain as they were in the Preform. They are all un-touchable, nugget-like stones. To be sure, there can be alterations in tense; in number (singular or plural); or in person (I, you, she, it, we, he, they). Other than that, all Preforms are like diamonds: they are fixed and free from the vicissitudes and transformations of normal syntax. Preforms are treated like the single words they have become. They refer to one thing and one thing only-the new semantic feature. For that reason, they are of enormous value in 
the study of English as a Second Language-and possibly other second languages taught in all countries of the globe. In an environment that abounds in multiplicity, the singularity of Preforms makes it possible to proceed.

\section{Juxtaposing Preforms}

All Preforms are consciously or subconsciously "memorized," thereby avoiding the almost impossible hassle of trying to assemble native-sounding phrases with a particular meaning. Almost always, the result of such an attempt is not understandable. Preforms offer speakers a rest, a respite from a grueling, often lost attempt to sound Native. Students can-on the other hand-juxtapose Preforms, offering them additional repose as they struggle with other problems of expression. Examples of Preforms used in this great easing follow. Preforms are written in italics below. Slash marks indicate separate Preforms.

\section{Memorizing Preforms}

Preforms become part of one's own personal repertoire through one's in-voluntary or voluntary committing them to memory. When teachers realize this, they can assign 5 or 10 Preforms to be memorized for the session that follows. Rarely does this present a problem: only in highly dubious educational theory is "memorization" frowned upon. With the invaluable assistance of illustration in one form or another on the internet, teachers can discuss these Preforms. Students can study with or without the computer. An immense motivating factor is the fact that Fully Native Speakers use a great many Preforms in their speech. In fact, everything the student learns in this exercise is immediately usable. Students are not memorizing declensions or strings of irrelevant random numbers: they can use the Preforms immediately with absolute certainty that the Preforms are 100\% correct-within their boundaries.

For example, "Day in and day out, she played hard to get." There is one meaning for "day in and day out," and that meaning is approximately ALWAYS. There is also one and only one meaning for "she played hard to get," and that meaning is approximately PRETENDED TO BE UNINTERESTED. I say "approximately" because there is no other way to capture the many subtleties of "she played hard to get." This seems to be one very important reason why Preforms exist in the first place: they help us express particular shades of meaning that cannot be expressed in any other way.

Preforms, however, are quite emphatically "social undertakings" and require-and receive-close, interpersonal cooperation. The minds of infants and teenagers are like sponges in the river of words they hear every day. It is not a matter of coming into contact with a pre-existing "master list" from one language or another; it is a matter of each person building up his or her own, individual list of words-and calling that list a French Dictionary; a Swahili Dictionary or a Russian Dictionary, complete with a carefully constructed guide to thinking and behaving in each culture.

There is some mechanism that registers heard Preforms with break-neck speed and is able to isolate and record them. If you attempt to create your own Preform, you will be making an enormous mistake. People 
Table 2 Juxtaposed Preforms

\begin{tabular}{|l|l|}
\hline 1 & Be that as it may, it's worth fixing. \\
\hline 2 & With eyes wide open, she saw the writing on the wall and knew she would be fired. \\
\hline 3 & They ran out the clock, and thereby did a number on their opponents. \\
\hline 4 & $\begin{array}{l}\text { The new evidence that the lawyer presented in court was like a smoking gun and } \\
\text { suddenly I didn't feel all that well. }\end{array}$ \\
\hline 5 & They have been head over heels in love for ump-teen years. \\
\hline 6 & He took it hook, line and sinker; nevertheless, let's give him a run for his money. \\
\hline 7 & It takes one to know one! \\
\hline 8 & So, now, it's off to the races! \\
\hline 9 & Her house is only a stone's throw from here, and likely we'll find her safe and sound. \\
\hline 10 & It has come to my attention that you've been running around with - shall we say - bad company. \\
\hline 11 & It's been a knock-down, drag-out fight, but she'll get over it, by and by. \\
\hline 12 & Let's get the show on the road. \\
\hline 13 & Get it while the getting's good! \\
\hline 14 & I slept through the wake-up call, and now I'm scared to death of the professor. \\
\hline 15 & Let's give her a big hand because she was right on the money in her predictions. \\
\hline 16 & What you see is what you get! \\
\hline 17 & We've virtually lost the game. We'll have to bite the bullet if that Hail-Mary pass fails. \\
\hline 18 & He's got a tiger by the tail! I kid you not! \\
\hline 19 & It's very hush-hush but she inherited the whole kit and caboodle. \\
\hline 20 & $\begin{array}{l}\text { No one wanted to point it out until Chuck came along. He always calls a spade, } \\
\text { a spade, and he said that this was an enormous case of mission creep. }\end{array}$ \\
\hline
\end{tabular}

might hear "day back and day forth," but they will not understand your meaning. You might make another attempt and say, "days in and days out," and they will again think that your English is very, very bad. Only by repeating precisely, "day in and day out," will they relax and completely understand. In communicating, two or more interlocutors must try to have complete and precise control of what they are saying, and of what is being said. In other words, Preforms help a particular language to become itself.

\section{Listen but Don't Repeat}

Above are some of the reasons why I consider Preforms to be the central structure in English, and probably in all verbal languages. The immediate benefit to students is apparent. They can construct a sentence, the Preform Part of which they can always be positive is $100 \%$ perfect. That is exactly the way Natives speak. 
There is no pre-existing body of rules and structures that serve as a model for Native Speakers as they are learning their first language. Instead all language is local, that is individual.

Another incredibly effective use of memorization is listening to-without repeating-a single 10-20-word sentence spoken by a Fully Native Speaker with a clear, slow voice and an accent you consider of a sufficiently high social status. Put the recorder-playback in a "repeat" mode and listen-don't speak-to the single 10 or 20-word sentence again and again for at least 10 minutes in the morning, 10 minutes the afternoon, and another 10 minutes at night. After a couple of days, your sub-conscious mind and intonation and vocal chords and lips and tongue-position will learn to make the second language sound far better than if you analyze it with your conscious mind or attempt to repeat what you hear one time. These organs will start making distinctions that you could not, and possibly still cannot hear.

It is fascinating but a fact that a great many Preforms are listed and explained on the internet. For example, look at "skip to my Lou, my darlin"" and "to a T" and type each in the search engine. Probably, you will be as fascinated as I was to see the richness of illustration. Learning to speak and write another language involves learning a great many details in the culture of that language: studying Preforms on the internet is invaluable in this and often constitutes an entire lesson.

\section{Proportions of Preforms}

Countless fixed and unchanging stretches of lexical items - Preforms - seem to occur in all areas of languagesongs, poems, short stories, operas, books, sporting events, political slogans, prayers, scripture, correspondence, speeches and conversations of all sorts. It is impossible for me to learn the actual number structures in these various areas that I would classify as Preforms. Nevertheless, it is important that I estimate the proportion of Preforms to Non-Preforms because of the great amount of time that I have spent studying these matters, and because of the importance of this issue to linguistics in general and to language teaching in particular. I have already called Preforms the "pinnacle" or lofty height among linguistic structures. I would also like to affirm my position on the crucial importance of memorization in acquiring these structures.

At present, this pedagogical use of memorization seems to be rarely employed-possibly because of the curious bias against overt memorization in contemporary education, and possibly because many linguists say that it is flat-out impossible to memorize a language. But that is precisely what Native Speakers of English do until they are about 15 years old-commit a great many Preforms $(50,000+)$ to memory. They "memorize" them and their cultural contexts-without thinking about it. Persons who have done this are likely, de facto, to be classified as Fully Native Speakers of English. They have in fact intentionally or unintentionally memorized a great many parts of their first language and culture. They have instant and incredibly precise access to most of the General Preforms in their first language-and usually, there is only one, single "first language." I emphasize this because often both of two languages come to be regarded as both qualifying as Native Languages. Both might well be spoken fluently, but rarely do both qualify as authentic first languages in which one person has equal control and access to most of the Preforms in that language. 
Possibly some linguists talk about the impossibility of memorizing a language under-estimate the capabilities of the human memory which are vast and unlimited in number. Consider the feat of medical doctors who often learn a whole dictionary of new terms. An M.D. who also specializes in law should have brains popping out of their ears-but they don't. They just continue to become wiser and to intake and process new information. It would be, in fact, interesting to consider the emergence of grammar and meaning as a result of the interaction of different parts of the human brain in the presence of Preforms. That, however, is a ball of wax that is for someone else-not me-to melt away.

The point at which a given set or stretch of words is Zapped into a Preform seems instantaneous and irreversible. The journey of that stretch can, however, often be distinguished before the final magical moment is reached. For example, "Performance hour" does not seem to constitute a Preform, although the needed transformation is nearby. "Show time!" with no verb, does seem to have been Zapped and transmuted.

\section{Preforms Can Be Taught}

Preforms might contribute to a useful index of verbal dexterity as well as proficiency, and can be concentrated on and taught in classroom-like settings. A Fully Native Speaker can benefit from such instruction by becoming better able to generate a wide range of Preforms such as "right on the money," "no stone left unturned," "plunge into despair," and "the die is cast." It might well be the case that such Preforms are not encountered until the individual is in his or her late teens, early twenties or even later. Apparently, there is no training in Advanced or even Regular Preforms offered anywhere in the world. Neither is the process of Preform Acquisition studied-anywhere. Which brings me to suggest that Preform Acquisition is sometimes a life-long process is never completely over and done with.

I suggest that an index of verbal dexterity be created and referred to as "Enhanced Fluency"(EF) of which scholarship seems to be largely unaware at present. I also suggest that related instruction be consciously evaluated and possibly developed for use in the classrooms of Non-Native as well as Native Speakers of English. The purpose of this step would be to make students and teachers aware of the advantages associated with the classification of Enhanced Native Speaker of English. One main advantage, it seems to me, would be in "discovering" a linguistic entity that can itself be measured and taught. Students everywhere should expect or ask for instruction in Preforms and Advanced Preforms.

This last point is based on the awareness that Preform instruction in a formal or informal classroom is quite possible. Professions that might particularly value verbal dexterity include teaching, writing, public speaking, public relations, and radio and television broadcasting. What led me to speculate thusly is encountering the speaking style of Rush Limbaugh, which seems to me to be rich in its use of Colloquial Preforms. Verbal dexterity is not only a demonstration of valuable local color skillfully employed, but a necessary part of a given speaker's meaningful and substantive statement. More generally, the Preform is an essential part of language, and as such must be taught as a discrete, objectively instantiated part of the developing lexicon. 


\section{Thousands of Classrooms}

Personally, in undergraduate school, I had two courses in French, two in German, and one in Greek. It is primarily me who is responsible for the lack of retention in my mind. I recall, however, no mention of the crucial concept of Preforms being made. Instead, it seems to have been thought that an analytical understanding of syntax and semantics in the presence of a foreign language would lead to something of lasting value to all concerned. As I recall, syntactic analysis and practical speech were conflated, with one effect being that little was learned. A few hundred Preforms by any name would have been most welcome.

A weak focus on grammar, a smattering of practical speech, plus a dearth of anything like Preforms seems to have been boiled in a pot and constituted a fundamental attitude, not only at my undergraduate school, but around the world in thousands of classrooms at all levels. Academic linguists were chasing foxlike subtleties of their field of language theory, with a sometimes justified, sometimes needless disdain of those whose interest was in language teaching. Admittedly, linguistics as it has evolved in the past-say 100 years-offered a meaty challenge. The core of language itself was being sought-and that is indeed intellectual activity at a very high and worthy level. But as it progressed, it came to be seen by some as a pursuit of smaller and smaller phenomena that are beyond the ability of human beings to comprehend. Any way you cut it, such a point is reached sooner or later by everyone on the face of the earth. That is to say that the Point of No Comprehension will be reached by all who delve into the relevant mysteries which lie eagerly in hiding. Of course, we must proceed at full steam pursuing what is not yet discovered.

\section{A Dreadful Error}

At some point, the study of linguistic theory seems to have unintentionally exerted undue influence on the study of applied linguistics, one of whose concerns was teaching English to a population of Non-Natives-or "English as a Second Language" (ESL). Although the number of class sessions in the latter far outnumbered the former, both are-in my opinion-highly respectable fields. Yet the study of linguistics proper has come to be considered by far the loftier of the two. Some members of some Departments of Applied Linguistics seemed to consider themselves deficient in not having an identity of their own. It seems to have been felt that the real scholars tended the mysterious fires that heated the cauldrons in which the brew of words was bubbling. According to this dreadful error, applied linguists simply report on a few of the evolving principles as they were developed by their cousins.

The misapprehension was possibly due to nomenclature. To refer to one field as "linguistics" and another as "applied linguistics" implies that the former is concerned with lofty principles, and that the other with the much lower application of those principles in the everyday world. Tilt! What hindsight tells us would have been far more accurate and not so prejudicial is, first, removal of the term "linguistics" from the bubbling brew. The former might have been called something like Structures in Communication and the latter, Education and Language, which could house ESL and other "Second Languages." This would also be more 
accurate in that the "Ph.D." is primarily concerned with formal research and writing for academic journals and the like; while the latter is on the edge of imaginative activities in the exploratory classroom. The fields are utterly different, and neither has more thunder and lightning than the other. It's like comparing the proverbial apples and oranges.

Before I retired in 2016, my colleague Dr. Mihri Napoliello collaborated in editing ten annual issues of The Journal of the Imagination in Language Learning. A few months before leaving New Jersey City University, I copied all of the pages of this temporarily discontinued publication and posted them on a dedicated website named CoreilImagination.com. More than 200 well received articles are still available there free of charge. My other website, Formulaics.com, presents more Preforms. When Mihri and I started the Journal, I grumbled about the names of the two fields referred to above, but my thoughts had not yet jelled. Now, they have. 